Received Date : 18-Dec-2015

Accepted Date : 25-Jan-2016

Article type : Letter

\title{
Low uptake of postpartum screening for Type 2 diabetes in women after a diagnosis of gestational diabetes
}

Postpartum screening rates for Type 2 diabetes after a diagnosis of gestational diabetes are low across many high-income countries, ranging from 20 to $58 \%$ up to 1 year after delivery [1,2]. Subsequent annual follow-up rates plateau at $20 \%$ [1].

We conducted an audit to assess postnatal diabetes screening rates in 233 women living in the city of Southampton with a history of gestational diabetes, diagnosed using WHO criteria. Current local practice involves giving verbal and written information regarding the risks of Type 2 diabetes after pregnancy at the initial consultation. After delivery and before discharge, all women are recommended to undergo postnatal glucose testing and are informed verbally and in writing to contact their general practitioner for annual ongoing screening. A copy of this letter is sent to the woman's general practitioner.

The women included in our audit were aged $30.6 \pm 5.2$ years, $42.9 \%$ were from an Asian ethnic background and $39.9 \%$ were white. The mean BMI was $29.6 \pm 7.2 \mathrm{~kg} / \mathrm{m}^{2}$, with $28.6 \%$ of the women being overweight and $25.6 \%$ being obese.

This article has been accepted for publication and undergone full peer review but has not been through the copyediting, typesetting, pagination and proofreading process, which may lead to differences between this version and the Version of Record. Please cite this article as doi: 10.1111/dme.13084

This article is protected by copyright. All rights reserved. 
In all, 229 women (98.3\%) had a fasting plasma glucose test before discharge to the community.

Three had impaired fasting glycaemia but normal results on re-testing. A total of 80 women (34.3\%) had glucose testing in the first year postpartum. The results for three women were consistent with a diagnosis of diabetes and another three women had impaired fasting glycaemia. Subsequently, $12.2 \%$ ( 16 of the 131 who were $\geq 2$ years postpartum) and $17.8 \%$ (eight of the 45 who were $\geq 3$ years postpartum) were screened 2 and 3 years after delivery, respectively. Two years after delivery, two women had elevated fasting plasma glucose levels and a further woman was diagnosed with Type 2 diabetes. Three years postpartum, one woman had impaired fasting glycaemia and another was diagnosed with Type 2 diabetes. Obese women were less likely to attend screening than women with a normal BMI $(P=0.011)$. Screening rates varied by postcode district from 22.5 to $50.0 \%$. There was no association between age, ethnicity, diabetes treatment during pregnancy or parity and the likelihood of glucose testing 1 year postpartum.

In contrast to the high immediate postnatal diabetes screening rates in hospital, the low long-term rates of postpartum screening in women with a history of gestational diabetes represents a missed opportunity to prevent diabetes and its complications.

The follow-up of gestational diabetes crosses the divide between primary and secondary care and involves two secondary care specialities, diabetes and obstetrics. A proportion of women may therefore be lost because of ambiguity between primary and secondary care responsibilities for postnatal screening [1]. Poor communication and a lack of agreed universal protocols across primary and secondary care have previously accounted for poor interdisciplinary co-ordination of care. Despite the discharge letters, the importance of screening within primary care may not be fully appreciated [3]. Local general practitioners have highlighted the large volume of correspondence from secondary care, which makes it easy to miss information. Providing an action request as a bullet point at the start of the letter or a separate 'action letter', emphasizing the importance of screening, could 
prompt the practice to add the woman to a practice-based gestational diabetes register and promote annual recall and review according to the National Institute for Health and Care Excellence guideline standards.

In South Australia, a Gestational Diabetes Mellitus Recall Register was set up that sends a reminder letter $\sim 15$ months after a women's expected delivery date that they should be screened for diabetes [4]. In 2009 , the scheme resulted in $56.3 \%$ of women on the register who returned the update form returning for postpartum screening [5]. A similar scheme in Ontario, Canada sends postal reminders to women and their doctors. This led to a screening increase to $60.5 \%$ (49/81 women), compared with $14.3 \%$ (5/35 women) where no reminder was sent [6]. Financial rewards through the Quality and Outcomes Framework may be needed to create a register and incentivize primary care to screen for diabetes.

Although we cannot ascertain the reasons for postcode differences in screening, this may reflect differences in deprivation or individual general practice policies. We do not know if women were invited and did not attend or whether there was no recall. High BMI was the only demographic factor associated with the uptake of screening. This may relate to hesitancy in this population to make lifestyle changes to manage their diabetes risk, or obesity may be a proxy marker for deprivation.

More than $40 \%$ of our cohort were from an Asian background, in whom language and communication may be a barrier to understanding information given by healthcare professionals [7]. Postnatal diabetes leaflets written in different languages may make the information accessible.

This article is protected by copyright. All rights reserved. 
It is possible that our estimates of screening are low because some women may have moved out of the city of Southampton and received screening elsewhere.

Our audit found low rates of annual postpartum screening and calls for improved communication between professionals and women from all backgrounds to raise awareness of the opportunity to prevent diabetes and its complications.

\author{
O. Adekojo ${ }^{1}$, K. R. Revell ${ }^{2}$, H. Preece ${ }^{2}$, S. Morris ${ }^{2}$, M. A. Coleman ${ }^{2}$ and R I G Holt ${ }^{1,2}$ \\ ${ }^{1}$ Human Development and Health Division, University of Southampton Faculty of Medicine and \\ ${ }^{2}$ University Hospital Southampton NHS Foundation Trust, Southampton, UK \\ Corresponding Author: r.i.g.holt@soton.ac.uk
}

\title{
References
}

1. McGovern A, Butler L, Jones S, van Vlymen J, Sadek K, Munro N et al. Diabetes screening after gestational diabetes in England: a quantitative retrospective cohort study. Br J Gen Pract 2014; 64 :e17-e23.

2. Ferrara A, Peng T, Kim C.Trends in postpartum diabetes and subsequent diabetes and impaired fasting glucose among women with histories of gestational diabetes mellitus: a report from the Translation of Research Into Action for Diabetes (TRIAD) Study. Diabetes Care 2009; 32: 269-274.

3. Wilkinson SA, Lim SS, Pennington A, O'Reilly SL, Asproloupos D et al. Who's responsible for the care of women during and after a pregnancy affected by gestational diabetes? Med J Aust 2014; 201: S78-S81.

This article is protected by copyright. All rights reserved. 
4. Chittleborough C, Caudle L et al. The Gestational Diabetes Mellitus (GDM) Recall Register Pilot Project Evaluation Report. Government of South Australia: Department of Health, 2005.

5. Chittleborough CR, Baldock KL, Taylor AW, Hague WM, Willson T, Martin W et al. Long-term follow-up of women with gestational diabetes mellitus: the South Australian Gestational Diabetes Mellitus Recall Register. Aust N Z J Obstet Gynaecol 2010; 50: 127-131.

6. Clark H, Graham I, Karovitch A, Keely EJ. Do postal reminders increase postpartum screening of diabetes mellitus in women with gestational diabetes mellitus? A randomised controlled trial. Am J Obstet Gynaecol 2009; 200: 634.e1-634.e7.

7. Sohal T, Sohal P, King-Shier KM, Khan NA. Barriers and Facilitators for type-2 diabetes management in south asians: a systematic review. PLoS One 10(9):e0136202.

This article is protected by copyright. All rights reserved. 